\title{
Independence of Changes in Behavior From Cognition and Function in Community-Dwelling Persons With Alzheimer's Disease: A Factor Analytic Approach
}

\author{
Rochelle E. Tractenberg, Ph.D., M.P.H., Myron F. Weiner, M.D., Jeffrey L. Cummings, M.D., \\ Marian B. Patterson, Ph.D., and Leon J. Thal, M.D. \\ Department of Biomathematics and Biostatistics, Georgetown University School of Medicine, \\ Washington, D.C.; the Departments of Psychiatry and Neurology, University of Texas \\ Southwestern Medical Center; Reed Neurological Research Center, University of California, Los \\ Angeles; Department of Psychiatry, University Hospitals of Cleveland; Alzheimer's Disease \\ Cooperative Study, Department of Neurosciences, University of California, San Diego.
}

\section{Abstract}

The authors' main objective was to investigate the relationship between changes in psychopathological, cognitive and activity of daily living (ADL) instrument scores over 12 months in community-dwelling persons with Alzheimer's disease (AD). A secondary objective was to evaluate the validity of dividing the Clinical Dementia Rating (CDR), a global dementia staging instrument into cognitive and functional subscores.

Changes in measures of psychopathology, cognition and function between the baseline and 12month visits were entered into these post hoc analyses of data from a one-year clinical trial to evaluate behavioral, cognitive and functional assessment instruments for use in clinical trials with $\mathrm{AD}$ patients. Exploratory factor analysis was used to determine whether there was independence between changes in any of these three domains of interest for this disease population; participants were a cohort of 187 well-characterized, community-dwelling persons with AD.

One-year change in the behavioral symptoms of this cohort of persons with AD was statistically independent from changes in scores on cognitive and functional measures. Some evidence of independence of 12 month changes in cognitive and functional measures was found. Cognitive and functional subscores for the CDR were supported. These findings suggest that changes in behavior and cognition in dementia may have distinct pathophysiologies.

\begin{abstract}
Alzheimer's disease (AD) progression is characterized by cognitive changes resulting from neuronal malfunction or death. These changes are accompanied by losses of functional ability as well as the development of behavioral symptoms. Neuroimaging and neuropathological studies suggest that behavioral and cognitive changes in dementia may be mediated by similar but not identical pathophysiological changes. ${ }^{1}$ If changes in behavior
\end{abstract}

Copyright $@ 2005$ American Psychiatric Publishing, Inc.

Address correspondence to Dr. Tractenberg, Department of Biomathematics and Biostatistics, 7 East Main, M702, Georgetown University Hospital, 3800 Reservoir Rd, NW, Washington, DC 20007; ret7@ georgetown.edu.. 
are due to different mechanisms of the disease process, and are not solely a consequence of cognitive changes, then symptoms in these domains should change independently over time.

In their 1996 review, Cummings and Kaufer ${ }^{2}$ found evidence for the contributions of cholinergic deficit to both the cognitive and behavioral symptoms of AD. Imaging studies of frontal lobe function/metabolism (not necessarily in persons with AD) have suggested two relatively independent functionalities (behavior and executive function) for different areas of the frontal lobes. ${ }^{3,4}$ Impaired executive function has been associated with significant neuropsychiatric symptomatology, such as depression, ${ }^{5}$ apathy, ${ }^{4,6}$ and agitation,,${ }^{7,8}$ but Sarazin et al. ${ }^{3}$ reported different relationships between executive dysfunction and behavioral symptomatology in different frontal lobe regions. Executive dysfunction represents an important neuropsychological entity 9,10 and can complicate the evaluation and diagnosis of persons with $\mathrm{AD} .{ }^{11}$ It has been argued that frontal lobe involvement in $\mathrm{AD}$ (but not frontotemporal dementia) may give rise to its characteristic, but not ubiquitous, behavioral disturbances. ${ }^{12}$ In addition, Royall and Polk ${ }^{12}$ have argued that it is the lack of involvement of the frontal lobes that differentiates dementias like AD from other dementias. This research has focused mainly on delusions, apathy and depression and although it has emphasized the neurophysiologic changes that accompany observed levels of symptomatology (behavioral, cognitive, or both), it has not included function nor been utilized to explore AD longitudinally.

Two recent studies support a relationship between changes in cognition and function in patients with AD. Cullum et al. ${ }^{13}$ found that cognitive scores strongly predict functional scores in persons with AD. A study by Tractenberg et al. ${ }^{14}$ provided preliminary evidence that behavioral symptoms (e.g., agitation, restlessness, and symptoms of psychosis, depression, paranoia) were more closely associated with changes in function scores than they were to changes in cognitive measures. Both studies suggest a close relationship between changes in cognition and function.

The present study was undertaken to test the hypothesis that cognitive and functional losses and changes in behavioral symptoms are associated. We sought to evaluate the underlying factor structure of changes in these domains using factor analysis, a technique by which the underlying structure of a particular set of variables can be evaluated. ${ }^{15,16}$

One possible outcome of factor analyses carried out on changes in the cognitive, functional, and behavioral domains is that changes in function and behavioral scores may associate (i.e., load on the same factor) and change in cognition has a separate "fundamental influence" (i.e., loads on a different factor). Another possibility is that cognitive and functional changes load on the same factor with behavioral changes loading on a separate factor. Each of these outcomes supports the conceptualization of a disease process with two main effects. Changes in all three domains could load on a single factor, suggesting a degree of dependence for all, or conversely, changes could load on three separate factors, suggesting independence of changes in all three domains. Any of these outcomes could have implications for conceptualizing the underlying pathophysiology and interventions as well as clinical trial design in this patient population. 
In this study, changes in score on well-known, valid instruments assessing cognitive status, functional ability, and behavioral symptoms were entered into a series of exploratory factor analyses. Additional change scores for cognition and function were included for greater representation of each domain.

\section{METHODS}

\section{Subjects}

The data examined in this post hoc analysis came from a multicenter clinical study (the English Instruments Study) of new clinical instruments for the assessment of AD patients. ${ }^{17}$ The English Instruments Study was conducted by the Alzheimer's Disease Cooperative Study, a large consortium of federally funded Alzheimer's Disease Centers. There were 27 participating sites. The study sample included 119 men and 187 women whose mean age was 71.9 years $(\mathrm{SD}=9)$. Almost all $(>95 \%)$ were Caucasian. All subjects met National Institute of Neurological and Communicative Disorders and Stroke and the Alzheimer's Disease and Related Disorders Association criteria ${ }^{18}$ for probable AD. Subjects with other brain diseases were excluded. Both presenile and senile onset cases were included. They were stratified for dementia severity ${ }^{17}$ by Mini-Mental State Exam (MMSE) ${ }^{19}$ score. Subjects were not receiving cognition-enhancing drugs, and only the subjects in the most severe groups (MMSE scores of 0-4, 5-9) were allowed psychotropic drugs ( $\mathrm{N}=7$ of roughly 100 persons in these groups). The only general medical exclusions were poor general health (e.g., progressive heart failure, active malignancy other than skin cancer, poorly controlled diabetes). Current or previous major psychiatric disorders were also exclusionary.

There were 242 individuals with diagnosis of probable AD recruited to the 12-month study. Seven participants were also enrolled in another study of interventions for behavioral symptoms in $\mathrm{AD}$ patients. These seven subjects were excluded from the present study because of their exposure to the behavioral interventions. Of the 235 subjects, 187 provided responses at both the baseline and 12-month visits. Subjects were evaluated at screening and at 6- and 12-month visits. Half of the cohort was randomly selected to return at 1- and 2month visits. The present evaluation focuses on the changes between the baseline and 12month visits in scores on measures of cognition, function (activities of daily living), and behavior. Multiple instruments were used to assess each of these domains, and this study focuses on four instruments representing these three domains (described below).

\section{Instruments}

The MMSE ${ }^{19}$ is an instrument used by clinicians to assess global cognitive functioning (see reference 20 for review of the MMSE and its characteristics). It is a 30-point scale with higher scores representing better cognitive functioning. Simple change in the MMSE score was calculated based on the first and last visit scores.

The Clinical Dementia Rating (CDR $)^{21,22}$ is an instrument developed for the staging of dementia and validated in persons with Alzheimer's disease. ${ }^{21}$ There are six domains: memory, judgment and problem solving, orientation, community activities, personal care, 
and home and hobbies. Each of these domains or "boxes" (each appears in a box on the rating sheet) is rated for the level of disability, with 0 representing normal (no disability), 0.5 representing questionable impairment (personal care does not have this rating), and 1, 2, and 3 representing mild, moderate and severe impairment, respectively. ${ }^{22}$ Ratings are derived by the clinician based on semistructured interviews with both the patient and the caregiver.

The CDR provides a global rating of dementia derived via algorithm using the memory score weighted according to the scores of the other boxes, or a sum of the individual box scores ("sum of boxes"). In this study, the sum of boxes was used as an indicator of global status. Additionally, the box scores were grouped according to whether they can be considered to represent cognitive (memory, judgment and problem solving, orientation) or functional (community activities, personal care, home and hobbies) domains (J. Morris, personal communication), and two subsums of box scores were calculated (cognition and function). The range for each subscore was $0-9$ with 0 being best. Change was calculated such that negative values represented worsening.

Calculated change in the global CDR is not as informative as changes in sums of box scores because the global rating is categorical and change in stage occurs slowly. Conversely, changes in ratings for any given box score, while indicating symptomatic change for the patient, do not automatically result in a change in the global CDR stage (see reference 22 for scoring rules). In this study, change in the total sum of boxes, as well as changes in the subsums of boxes for the cognitive and functional domains were calculated and included in the analyses.

A secondary aim of this analysis was to evaluate the validity of the cognitive/functional subscores of the CDR. This delineation would be supported if changes in box scores characterized as "cognitive" were observed to associate strongly with change in MMSE and if changes in box scores characterized as "functional" were observed to associate strongly with change in activities of daily living scores.

The Alzheimer's Disease Cooperative Study activities of daily living (ADL) inventory 23 (appendix 1) is a set of 23 activities (e.g., dressing oneself, using the phone, preparing meals), which are rated by the caregiver according to the amount of assistance the patient/ subject requires to carry out each. Scores on this instrument range from 0 to 78 with higher scores indicating greater degrees of assistance required. The amount of change in ADL score per year in patients with $\mathrm{AD}$ is not documented. Changes from baseline (losses of function) were calculated for this analysis. Under the English Instruments Study protocol, a 38-item ADL inventory was administered. ${ }^{23}$ The 38 items were culled from several ADL inventories representing important areas of function in which persons with $\mathrm{AD}$ might show impairment over the course of the disease. Galasko et al. ${ }^{23}$ carried out an analysis of these 38 items and reported that 23 of them were most sensitive and representative for this patient population.

The responses to these 23 items were recoded and re-scored to represent the current version of this ADL inventory (D. Galasko, personal communication), which has a maximum (best) score of 78 points. Change was calculated such that negative values represented worsening. 
The Consortium to Establish a Registry for Alzheimer's Disease Behavior Rating Scale for Dementia (BRSD) ${ }^{24,25}$ is a set of 46 behavioral items assessing anxiety, depression, agitation, psychosis, hallucinations, and other psychopathology common in persons with dementia. The symptoms are rated for their frequency during the previous month by the caregiver or knowledgeable informant. One item is general ("anything else?"), and emergence on this item is not calculated. Eight items are not rated for frequency but rather as yes/no ("ever/never since dementia began") and absence at baseline and presence later represents emergence for these items. Total BRSD scores range from 0 to 156, with higher scores representing greater levels of behavioral disturbance. The BRSD was developed specifically for persons with dementia and was found to have good reliability and validity in the cohort studied in the present report ${ }^{26}$ and current scoring rules are described fully in Mack et al. ${ }^{27}$

In this study, changes in behavior were evaluated in two ways. Change in the total BRSD score was calculated to ascertain change in the overall level of disturbance ("simple change"). The emergence of new symptoms was also calculated. Emergence is defined as the ratio of items that were rated absent or minimally frequent at the baseline visit and that were rated present at the last visit divided by the number of items which were eligible to emerge (rated absent or minimally frequent at the baseline visit). This calculation is described in detail elsewhere. ${ }^{28,29}$

The denominator of the emergence rate is the number of BRSD items which were eligible to emerge at baseline; the numerator is the number of items which were eligible and did emerge by the later visit (12 months). Scores were not imputed; therefore, if one or both items required for determining emergence was missing (either due to missed visit or to scores set to zero), then that item's emergence value was missing and it was neither counted as emerged nor eligible to emerge. Emergence values greater than zero represent worsening (emergence cannot be negative).

For each subject, the emergence rate was calculated and used in this study as an indication of worsening psychopathology. This measure of behavioral change has been found to be more sensitive than the change in total BRSD score ${ }^{29}$ to changes observed in this population. These two behavioral change scores were expected to be associated because they were derived from the same instrument. However, the simple change score includes both improvement and worsening over all items, while emergence rate represents only worsening (i.e., only those items which were absent/minimally present at baseline and present later). Therefore, the association between simple change and emergence was not expected to be strong.

Also included in these analyses were patients' age and years of education.

\section{Statistical Methods}

Change in MMSE, ADL, BRSD total score, CDR total sum of boxes, and cognitive and functional subsums of boxes scores were calculated so that negative scores reflected worsening except for BRSD total, on which positive change indicates worsening. 
Emergence rate is itself a measure of change, so change in emergence was not calculated. Emergence is always positive, indicating worsening.

The analysis proceeded in a stepwise manner, and all procedures were carried out in SAS Version 8 for Windows. ${ }^{30}$ First, correlations (Pearson's) between the change scores were calculated. It was anticipated that, for example, changes in the subsum of box scores would correlate with change in the overall sum of box scores. Emergence and simple change represent different types of change calculation; however, both were calculated based on BRSD items (emergence) or total scores (change), and it was anticipated that change in total BRSD would correlate to some degree (although possibly not a high degree ${ }^{29}$ with emergence rate.

Principal components analyses, extracted from the correlation matrix, were carried out to estimate the eigenvalues for each factor and to determine the proportion of variance accounted for by the factor solutions in the series.

Two series of factor solutions were then evaluated via factor analysis. The number of factors retained in the first solution (three) was determined by the competing hypotheses about the relationships between the domains under study: a minimum of three factors would be required to allow changes in each domain to load on a separate factor. The number retained in the last solution evaluated was determined by both total variance accounted for and the makeup of the factors themselves (i.e., when solutions stopped making sense or became inconsistent). The main goal of factor analysis is to derive fewer factors than one has variables to start with, and there were 5-6 scores plus two demographic variables in the present analysis. Therefore, six or fewer factors would be desirable. Varimax rotation was employed to facilitate the interpretability of the factor solutions.

Comrey and Lee ${ }^{31}$ describe factor loadings of 0.55 to represent "good" interpretive value (p. 243 ), so the cutoff for concluding that a variable "loads" on a factor was a loading of \pm 0.55 on that factor, and if variables loaded on more than one factor, the factor with the higher loading was the one on which the variable was considered to have "loaded." The series of factor solutions were evaluated to determine if there was evidence of consistent relationships among the domains. The first series included change in CDR sum of boxes and excluded changes in the CDR subsums of boxes. The second series excluded change in the total sum of boxes and included changes in the subsums of boxes.

Correlations between age, education and changes in all scores were computed, as were descriptive statistics. Principal components analysis was carried out, and series of factor analyses with varimax rotation were carried out.

\section{RESULTS}

Table 1 contains the descriptive statistics for the variables of interest in this cohort.

Correlations among the variables were not strong (relative strengths as defined by Cohen and Holliday). ${ }^{32}$ Table 2 contains the correlations among the cognitive and functional change scores and emergence of psycho-pathology (i.e., correlations for age and education 
do not appear). The correlation between emergence and change in ADL ( $\mathrm{r}=-0.262$ "low") was stronger than that between emergence and change in MMSE ( $r=-0.085$, "very low"). Similar associations were observed between emergence and changes in the functional and cognitive sums of boxes, respectively (both "very low"), as well as for change in BRSD and these four change scores (all "very low"). We observed positive correlations between changes in function and both global cognitive variables (ADL and MMSE: $r=0.407$; ADL and CDR sum of boxes: $r=0.502$; both "modest"). $P$ values for correlations were not computed since the correlations were used only to facilitate the analysis, not as outcomes.

Changes in the CDR sum of boxes and in the MMSE correlated positively ( $\mathrm{r}=0.458$, "modest"), and the change in ADL correlated well with change in the sum of functional boxes ( $\mathrm{r}=0.524$, "modest"). Changes in both the cognitive and functional subsums of boxes correlated to the same extent with changes in MMSE (both $r=0.447$ ), slightly stronger than the association between changes in MMSE and in ADL ( $\mathrm{r}=0.407)$ (all "modest").

\section{Including CDR Sum of Boxes}

The first series of factor analyses included the change in CDR total sum of box scores and excluded changes in the CDR subsums of boxes. Eigenvalues based on the correlation matrix showed that there were three factors with eigenvalues greater than one; these accounted for $68.4 \%$ of the variance in the sample. Stevens ${ }^{33}$ suggested that $75 \%$ represents "most" of the variance; four factors accounted for $79.9 \%$ of the variance, while five factors accounted for $87.7 \%$. The six-factor solution, accounting for $94.8 \%$ of the variance, was evaluated (see below) but was considered to be only of marginal interest since there were only seven variables in these analyses (there is limited desirability in separating seven variables into six factors).

Table 3 provides the loadings of the variables on each factor in each of the three factor solutions evaluated. In the three-factor solution (after varimax rotation), factor 1 comprised ADL, MMSE, and CDR Sum; factor 2 contained BRSD and emergence; and age and education made up factor three.

The four-factor solution (after varimax rotation) yielded the same first two factors as were observed in the three-factor solution (ADL/MMSE/CDR sum and BRSD/emergence). Factor 3 contained only age, and factor 4 contained only education.

Factor 1 of the five-factor solution (after varimax rotation) contained BRSD and emergence; factor 2 comprised ADL and CDR Sum; and factors 3, 4, and 5 were made up of just education, age, and MMSE, respectively.

\section{Including CDR Subsums of Boxes}

The second series of factor analyses included changes in the CDR subsums of boxes and excluded the change in CDR total sum of box scores. Eigenvalues based on the correlation matrix showed that there were three factors with eigenvalues greater than 1 ; these accounted for $64.2 \%$ of the variance in the sample; four factors accounted for $74.3 \%$ of the variance, and five factors $(82.9 \%)$ were needed to meet Stevens' criterion. 
Table 4 provides the loadings of the variables on each factor in each of the three factor solutions evaluated. After varimax rotation, the three-factor solution yielded the grouping of factor 1, comprising ADL, cognition, function, and MMSE; factor 2 contained BRSD and emergence; and age and education made up factor 3.

The four-factor solution (after varimax rotation) yielded the same first two factors as were observed in the three-factor solution (ADL/MMSE/cognition and function sums of boxes and BRSD/emergence). Factor 3 contained education, and factor 4 contained age.

Factor 1 of the five-factor solution (after varimax rotation) contained BRSD and emergence; factor 2 comprised ADL and CDR function subsum; factor 3 contained CDR cognitive subsum and MMSE. Factors 4 and 5 were made up of education and age, respectively.

\section{DISCUSSION}

Two series of post hoc exploratory factor analyses were carried out on changes in cognitive, functional and behavioral scores collected in a study of community-dwelling persons with AD. The analyses demonstrated that changes in behavior (as represented by simple change in total score and emergence) were statistically independent from changes in scores on cognitive and functional instruments.

Although modest separation of changes in cognitive and functional scores was observed, the results suggest that changes in these domains are closely related. We also observed that change in CDR box scores representing function loaded with changes in ADL and change in CDR box scores representing cognition loaded with changes in MMSE. While not definitive, this suggests that the characterization of the grouping of CDR box scores outlined in our analysis is reasonable.

Change in BRSD and emergence loaded together in each of three factor solutions in two series of analyses. These scores never loaded on factors containing scores representing change in cognitive and functional scores, suggesting that the causes of behavioral symptoms differ from those of cognitive and functional losses. The observed association between changes in function and in behavioral change (Table 2) may reflect a common mediating factor (such as frontal lobe dysfunction), while their failure to load on the same factor supports the suggestions that they have distinct pathophysiologies. This contrasts with the close relationship between cognition and function.

It was also observed that changes in cognitive and functional scores loaded together on the same factor (three- and four-factor solutions) or on two (five-factor solution) factors. The observed separation of the two functional and two cognitive change scores in the five-factor solution suggests that there may be some degree of independence of symptoms in these two domains, and future studies may address this possibility.

Limitations of this study must be considered. Primary among these is that there is strong evidence that the underlying cause of behavioral symptoms in AD is loss of executive function (see, e.g., reference 12) and no measure of frontal lobe or executive function was 
included in this study. Thus, the possible role of the frontal lobes in mediating behavior and function could not be investigated.

Similarly, we included only two measures of change in each domain, with the same instrument (BRSD) generating two representations of behavioral change. This decision arose from the fact that the BRSD was the only broad behavioral measure utilized in the study. Other instruments were considered, but their symptom lists were too specific (e.g., depression, agitation) to include in these analyses. The instruments employed all have documented reliability and validity in this disease population, particularly longitudinally, which supports their use in these analyses.

There are also several statistical issues. Stevens ${ }^{33}$ points out that factor analyses on samples smaller than 100 are problematic and characterizes sample sizes of 200-300 as "fairly large" (p. 370); he also notes that if the sample size is "large" compared to the number of variables in the analysis (N-to-variables ratio >25) then interpretation of the factors will be supported (p. 371). The present sample can be said to meet both of these criteria with a sample size of 187 and $\mathrm{N}$-to-variables ratio of at least 23 . An additional statistical concern is that missing responses and dropouts were not accounted for. BRSD scoring can be invalidated (leading to missing scores) if the caregiver/rater is unable to comment on the frequency of at least 12 items. Because these were exploratory analyses, we feel that the findings are tenable in spite of these limitations. Also, the analytic results met the criterion for "excellent" interpretability (i.e., all loadings were greater than \pm 0.71 ). ${ }^{31}$

If statistical requirements can be considered to have been met in these analyses, the makeup of the cohort may still limit generalizability of the results. The participants were communitydwelling patients capable of undergoing interpretable assessment at both of two visits 12 months apart. Also, patients with significant psychiatric symptoms were excluded from the study. Therefore, individuals with very severe dementia or behavioral symptomatology were not included in our analyses. Further, our two estimates of changes in behavioral disturbance levels were based on responses to items on a single rating instrument.

Participants ranged in dementia severity levels; the relationship between changes in cognition, function and behavior may be different for individuals at different stages of the disease. We observed relatively little change in any domain over the period of the study (Table 1), which could reflect the stability of the symptoms in this particular cohort or limited sensitivity to change over 12 months of the instruments used. Similar analyses involving change over longer periods, and with larger cohorts at each severity level might increase our understanding of the dynamics between symptoms in these areas. The CDR box scores might be useful in future longitudinal analyses as either an indicator of global status or broken down into functional and cognitive subsets, or both.

Changes in cognitive and functional scores over 12 months in this cohort are likely due to the same disease mechanism. Although one of the factor solutions suggested a degree of independence, the correlations (Table 2) among the change scores in cognitive and functional domains suggest dependence. The two demographic variables, age and education, did not correlate (Table 2). They loaded on separate factors in the four- and five-factor 
solutions in both series, and probably cannot be considered together as a combined "demographic" variable in future analyses.

Thus, despite earlier findings that changes in behavior may be more closely correlated with changes in function than with changes in cognition over 12 months in this cohort, this effort to better describe how symptoms in different domains change over 1 year in persons with $\mathrm{AD}$ reinforces recent findings $\mathrm{s}^{34}$-37 that changes in behavior are, to some extent, independent of changes in the other two domains. In spite of methodological limitations, our tentative results suggest that further analysis of the relationships between changes in these domains is warranted and these are under way in independent cohorts of persons with AD.

The relative independence of changes in behavioral from changes in cognitive symptoms in this cohort suggests partially independent mechanisms, and that both cognitive and behavioral symptoms are direct results of the disease process. Whether each results from different biochemical or anatomic lesions is not known, but it may be that differing combinations of behavioral and cognitive symptoms will point to subtypes of disease or will require different combinations of treatment modalities. For example, hallucinations occurring in persons with relatively intact cognition might be treated differently from the hallucinations that occur in persons with moderate severity of cognitive deterioration. The former might respond to anticholinesterases; the latter to anti-psychotics.

Variable patterns of neuronal death and neurotransmitter imbalances that account for differing cognitive presentations of $\mathrm{AD}$ (e.g., aphasia versus apraxia) may also account for presentations in which behavioral symptoms are more or less prominent than cognitive symptoms. The partial amelioration of behavioral symptoms in response to augmentation of cholinergic function ${ }^{38,39}$ points to a certain degree of overlap between these symptomatic areas, but the relationship is modest at best. ${ }^{39,40}$ It may be that certain agents for treatment of $\mathrm{AD}$ will have a differential effect on behavioral symptoms that would be undetected if only cognitive or functional measures were used to assess outcome. Therefore, in clinical trials, these domains and responses to interventions in each must be assessed completely and separately.

\section{Acknowledgments}

The authors wish to thank Dr. Anthony Gamst for invaluable assistance with the statistical methodology of this paper.

This study was supported by grant AG-10483 from the National Institute on Aging.

\section{References}

1. Finkel SI. Behavioral and psychological symptoms of dementia: a current focus for clinicians, researchers, and caregivers. J Clin Psychiatry. 2001; 62(suppl 21):3-6. [PubMed: 11584987]

2. Cummings JL, Kaufer D. Neuropsychiatric aspects of Alzheimer's disease: the cholinergic hypothesis revisited. Neurology. 1996; 47:876-883. [PubMed: 8857712]

3. Sarazin M, Pillon B, Giannakopoulos P, et al. Clinicometabolic dissociation of cognitive functions and social behavior in frontal lobe lesions. Neurology. 1998; 51:142-148. [PubMed: 9674793]

4. Stuss DT, Levine B. Adult clinical neuropsychology: lessons from studies of the frontal lobes. Annu Rev Psychol. 2002; 53:401-433. [PubMed: 11752491] 
5. Lockwood KA, Alexopoulos GS, van Gorp WG. Executive dys-function in geriatric depression. Am J Psychiatry. 2002; 159:1119-1126. [PubMed: 12091189]

6. Mendez MF, Perryman KM. Neuropsychiatric features of frontotemporal dementia: evaluation of consensus criteria and review. J Neuropsychiatry Clin Neurosci. 2002; 14:424-429. [PubMed: 12426410]

7. Litvan, I. Personality and behavioural changes with frontal-sub-cortical dysfunction. In: Lichter, DG.; Cummings, JL., editors. Frontal-Subcortical Circuits in Psychiatric and Neurological Disorders. Guilford; New York: 2001. p. 151-162.

8. Tekin S, Mega MS, Masterman DM, et al. Orbitofrontal and anterior cingulate cortex neurofibrillary tangle burden is associated with agitation in Alzheimer disease. Ann Neurol. 2001; 49:355-361. [PubMed: 11261510]

9. Royall DR, Lauterbach EC, Cummings JL, et al. Executive control function: a review of its promise and challenges for clinical research: a report from the Committee on Research of the American Neuropsychiatric Association. J Neuropsychiatry Clin Neurosci. 2002; 14:377-405. [PubMed: 12426407]

10. Elliot R. Executive functions and their disorders. Br Med Bull. 2003; 65:49-59. [PubMed: 12697616]

11. Royall DR. Executive cognitive impairment: a novel perspective on dementia. Neuroepidemiology. 2000; 19:293-299. [PubMed: 11060503]

12. Royall DR, Polk M. Dementias that present with and without posterior cortical features: an important clinical distinction. J Am Geriatr Soc. 1998; 46:98-105. [PubMed: 9434673]

13. Cullum CM, Saine K, Davis-Chan L, et al. A performance-based instrument to assess functional capacity in dementia: the Texas Functional Living Scale. Neuropsychiatr Neuropsychol Behav Neurol. 2001; 14:103-108.

14. Tractenberg RE, Weiner MF, Patterson MB, et al. Emergent psychopathology in AD patients over 12 months associated with functional, not cognitive, changes. J Geriatr Psychiatry Neurol. 2002; 15:110-117. [PubMed: 12083593]

15. Afifi, AA.; Clark, V. Computer-Aided Multivariate Analysis. 3rd ed.. Chapman \& Hall; Boca Raton, Fla: 1997.

16. Cudeck, R. Exploratory factor analysis. In: Tinsley, HEA.; Brown, SD., editors. Handbook of Applied Multivariate Statistics and Mathematical Modeling. Academic Press; San Diego: 2000. p. 266-296.

17. Ferris SH, Mackell JA, Mohs R, Schneider LS, Galasko D, White-house PJ, Schmitt FA, Sano M, Thomas RG, Ernesto C, Grundman M, Schafer K, Thal LJ. A multicenter evaluation of new treatment efficacy instruments for Alzheimer's disease clinical trials: overview and general results: the Alzheimer's Disease Cooperative Study. Alzheimer Dis Assoc Disord. 1997; 11(suppl 2):S1S12. [PubMed: 9236947]

18. McKhann G, Drachman D, Folstein M, et al. Clinical diagnosis of Alzheimer's disease: report of the NINCDS-ADRDA Work Group under the auspices of the Department of Health and Human Services Task Force on Alzheimer's Disease. Neurology. 1984; 34:939-944. [PubMed: 6610841]

19. Folstein MF, Folstein SE, McHugh PR. "Mini-Mental State": a practical method for grading the cognitive state of patients for the clinician. J Psychiatr Res. 1975; 12:189-198. [PubMed: 1202204]

20. Lezak, MD. Neuropsychological Assessment. 3rd ed.. Oxford University Press; New York: 1995.

21. Hughes CP, Berg L, Danziger WL, et al. A new clinical scale for the staging of dementia. Br J Psychiatry. 1982; 140:566-572. [PubMed: 7104545]

22. Morris JC. The Clinical Dementia Rating (CDR): current version and scoring rules. Neurology. 1993; 43:2412-2414. [PubMed: 8232972]

23. Galasko D, Bennett D, Sano M, et al. An inventory to assess activities of daily living for clinical trials in Alzheimer's disease: The Alzheimer's Disease Cooperative Study. Alzheimer Dis Assoc Disord. 1997; 11(suppl 2):S33-S39. [PubMed: 9236950]

24. Tariot PN, Mack JL, Patterson MB, et al. Behavioral Pathology Committee of the Consortium to Establish a Registry for Alzheimer's Disease: The Behavior Rating Scale for Dementia of the 
Consortium to Establish a Registry for Alzheimer's Disease. Am J Psychiatry. 1995; 152:1349_ 1357. [PubMed: 7653692]

25. Mack, JL.; Patterson, MB. Manual: CERAD Behavior Rating Scale for Dementia. 2nd ed.. Consortium to Establish a Registry for Alzheimer's Disease; Durham, NC: 1996.

26. Patterson MB, Mack JL, Mackell JA, et al. A longitudinal study of behavioral pathology across five levels of dementia severity in Alzheimer's disease: the CERAD Behavior Rating Scale for Dementia: the Alzheimer's Disease Cooperative Study. Alzheimer Dis Assoc Disord. 1997; 11(suppl 2):S40-S44. [PubMed: 9236951]

27. Mack JL, Patterson MB, Tariot PN. The Behavior Rating Scale for Dementia (BRSD): development of test scales and presentation of data for 555 individuals with Alzheimer's disease. $\mathrm{J}$ Geriatr Psychiatry Neurol. 1999; 12:211-223. [PubMed: 10616870]

28. Tractenberg RE, Jin S, Patterson M, et al. Qualifying change: a method for defining clinically meaningful outcomes of change score computation. J Am Geriatr Soc. 2000; 48:1478-1482. [PubMed: 11083327]

29. Tractenberg RE, Gamst A, Thomas RG, et al. Investigating emergent symptomatology as an outcome measure in a behavioral study of Alzheimer's disease. J Neuropsychiatry Clin Neurosci. 2002; 14:303-310. [PubMed: 12154155]

30. SAS for Windows, version 8.1. SAS Institute; Cary, NC: 2001.

31. Comrey, AL.; Lee, HB. A First Course in Factor Analysis. 2nd ed.. Lawrence Erlbaum Associates; Hillsdale, NJ: 1992.

32. Cohen, L.; Holliday, M. Practical Statistics for Students: An Introductory Text. Paul Chapman Publishing; London: 1996.

33. Stevens, J. Applied Multivariate Statistics for the Social Sciences. 3rd ed.. Lawrence Erlbaum Associates; Mahwah, NJ: 1996.

34. Tekin S, Cummings JL. Frontal-subcortical neuronal circuits and clinical neuropsychiatry: an update. J Psychosom Res. 2002; 53:647-654. [PubMed: 12169339]

35. Devanand DP, Brockington CD, Moody BJ, et al. Behavioral syndromes in Alzheimer's disease. Int Psychogeriatr. 1992; 4(suppl 2):161-184. [PubMed: 1288661]

36. Marin DB, Green CR, Schmeidler J, et al. Noncognitive disturbances in Alzheimer's disease: frequency, longitudinal course, and relationship to cognitive symptoms. J Am Geriatr Soc. 1997; 45:1331-1338. [PubMed: 9361658]

37. McCarty HJ, Roth DL, Goode KT, et al. Longitudinal course of behavioral problems during Alzheimer's disease. J Gerontol A Biol Sci Med Sci. 2000; 55:M200-M206. [PubMed: 10811149]

38. Cummings JL. Cholinesterase inhibitors: a new class of psycho-tropic compounds. Am J Psychiatry. 2000; 157:4-15. [PubMed: 10618007]

39. Kaufer DI, Cummings JL, Christine D. Effect of tacrine on behavioral symptoms in Alzheimer's disease: an open-label study. J Geriatr Psychiatry Neurol. 1996; 9:1-6. [PubMed: 8679057]

40. Weiner MF, Martin-Cook K, Foster BM, et al. Effects of donepezil on emotional/behavioral symptoms in Alzheimer's disease patients. J Clin Psychiatry. 2000; 61:487-492. [PubMed: 10937606] 


\section{TABLE 1}

Descriptive Statistics for Age, Education, and Change in Variables ${ }^{a}$

\begin{tabular}{lcrl}
\hline Variable & Mean & SD & Interpretation \\
\hline Age (years) & 71.5 & 8.9 & Age at intake (years) \\
Education & 13.3 & 2.9 & Years of education completed \\
Change in MMSE score & -3.9 & 4.2 & Loss of cognitive ability \\
Change in CDR & & & \\
Sum of all boxes & 2.3 & 2.1 & Increase of dementia level \\
CDR cognitive subsum of boxes & -1.1 & 1.2 & Loss of cognitive ability \\
CDR functional subsum of boxes & -1.2 & 1.3 & Loss of functional ability \\
Change in ADL score & -9.9 & 9.6 & Loss of functional ability \\
Change in BRSD score & 1.4 & 16.1 & Increase in behavioral symptoms \\
Emergence & & & $10.2 \%$ of possible emergence occurred \\
\hline
\end{tabular}

$\mathrm{SD}=$ standard deviation

${ }^{a}$ MMSE $=$ Mini-Mental Status Exam; CDR = clinical dementia rating; ADL = activities of daily living; BRSD = Behavior Rating Scale for Dementia

$b$ emergence over 12 months: on average, $10.2 \%$ of items absent or minimally present at baseline were rated present at the 12 -month visit 


\section{TABLE 2}

Correlations Between the Change Scores ${ }^{a}$

\begin{tabular}{|c|c|c|c|c|c|c|}
\hline \multirow[b]{2}{*}{ Area of Change } & \multicolumn{6}{|c|}{ Correlation Coefficient for Areas of Change } \\
\hline & ADL Score & BRSD Total Score & CDR Cognitive Subsum & Emergence & CDR Functional Subsum & MMSE Score \\
\hline BRSD total score & -0.165 & & & & & \\
\hline $\begin{array}{l}\text { CDR cognitive } \\
\text { subsum of boxes }\end{array}$ & 0.320 & -0.032 & & & & \\
\hline Emergence & -0.262 & 0.580 & -0.098 & & & \\
\hline $\begin{array}{l}\text { CDR functional } \\
\text { subsum of boxes }\end{array}$ & 0.524 & -0.137 & 0.447 & -0.159 & & \\
\hline MMSE score & 0.407 & 0.041 & 0.447 & -0.085 & 0.339 & \\
\hline Sum of all boxes & 0.502 & -0.103 & 0.832 & -0.032 & 0.868 & 0.458 \\
\hline
\end{tabular}

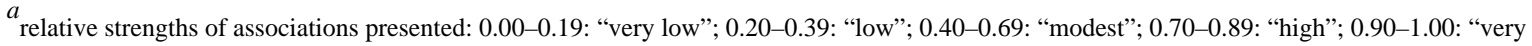
high" 32 ADL = activities of daily living; BRSD = Behavior Rating Scale for Dementia; CDR = clinical dementia rating; MMSE = Mini-Mental Status Exam 


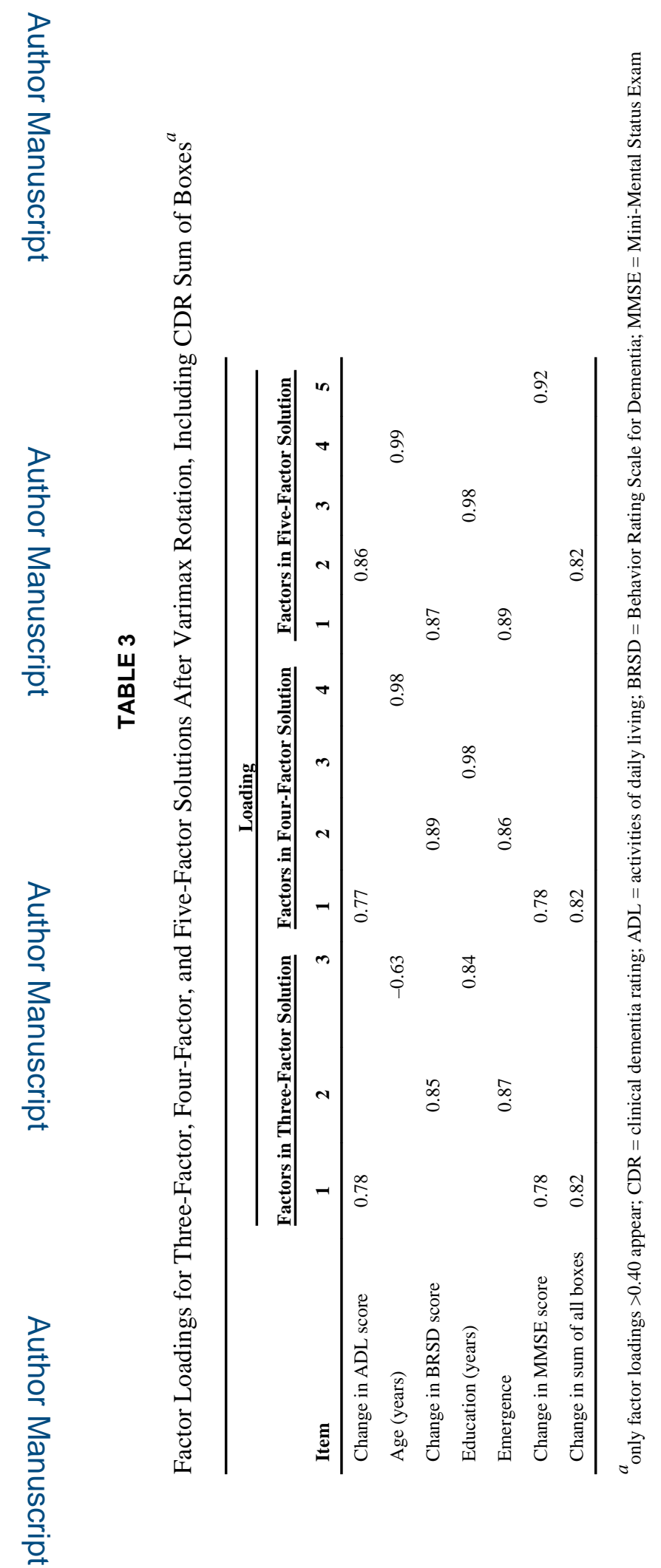

J Neuropsychiatry Clin Neurosci. Author manuscript; available in PMC 2015 March 29. 


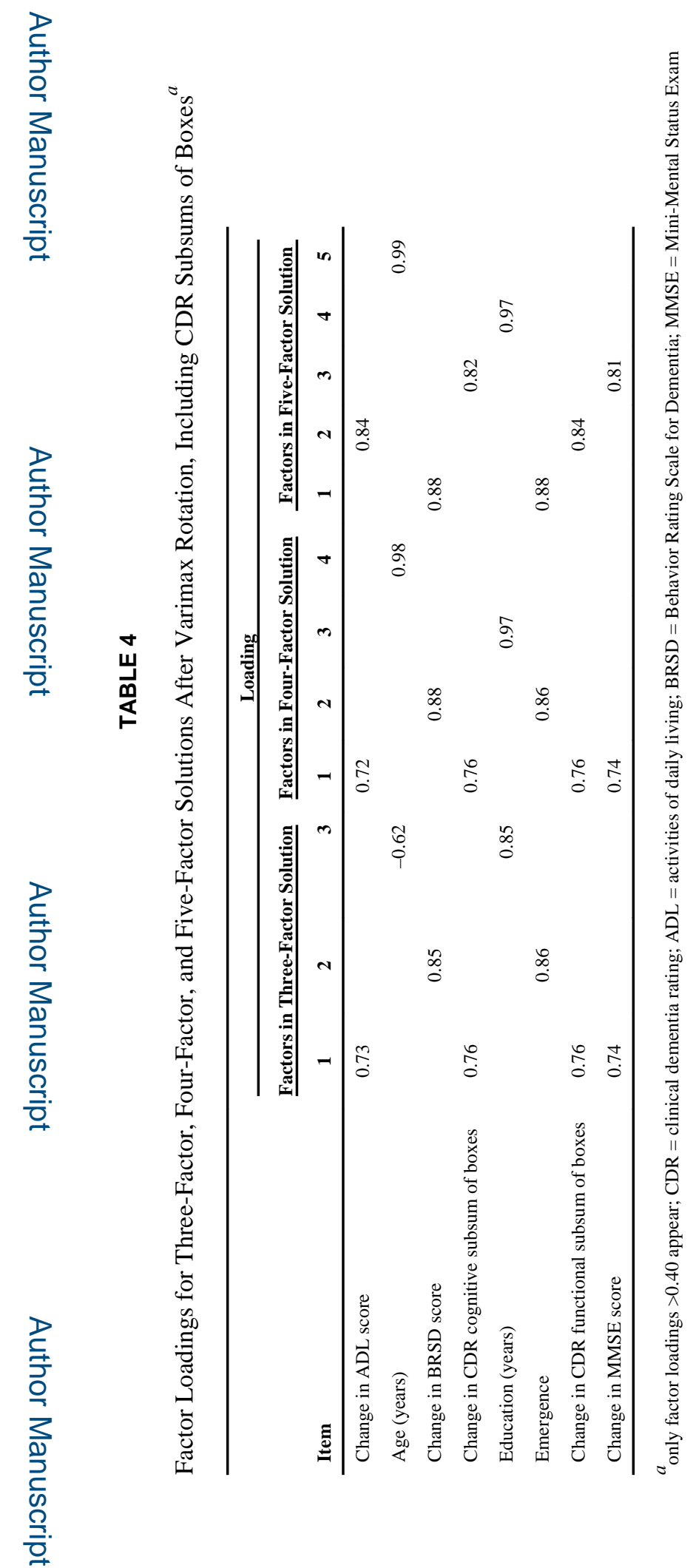

J Neuropsychiatry Clin Neurosci. Author manuscript; available in PMC 2015 March 29. 